\title{
Differentiating between fertilized and unfertilized eggs prior to incubation based on oxygen flux measurement
}

\author{
Wang Qiaohua ${ }^{1,2}$, Fu Dandan ${ }^{1}$, Ma Meihu ${ }^{2,3^{*}}$, Zhang Tao ${ }^{1}$ \\ (1. College of Engineering, Huazhong Agricultural University, Wuhan 430070, China; \\ 2. National Research and Development Center for Egg Processing, Huazhong Agricultural University, Wuhan 430070, China; \\ 3. College of Food Science and Technology, Huazhong Agricultural University, Wuhan 430070, China)
}

\begin{abstract}
One unresolved challenge in the egg industry is how to efficiently and non-invasively detect unfertilized eggs prior to incubation. This detection ability would not only significantly improve hatching rates and reduce costs but also conserve incubator space and prevent poor-quality embryos from contributing to the spread of infections. This study demonstrates a procedure for distinguishing between fertilized and unfertilized eggs prior to incubation by studying the respiratory differences between fertilized and unfertilized eggs using the Non-invasive Micro-test Technique (NMT). A customized micro-testing examination platform, NMT Egg Testing System (NMT-ETS) was constructed for the real-time monitoring of the intensity and rate of oxygen exchange between the egg and its external environment. The results from this study revealed that at room temperature, there is a significant difference in gas exchange rates between fertilized and unfertilized eggs. The results indicate that the oxygen flux of fertilized eggs exceed $20 \mathrm{pmol} /\left(\mathrm{cm}^{2} \cdot \mathrm{s}\right)$, whereas unfertilized eggs show a much lower oxygen flux. Based on the results, the NMT method can be used to effectively distinguish between fertilized and unfertilized chicken eggs.
\end{abstract} Keywords: oxygen flux, gas exchange rates, fertilized eggs, unfertilized eggs, non-invasive micro-test technique DOI: $10.25165 /$ j.ijabe.20171004.2606

Citation: Wang Q H, Fu D D, Ma M H, Zhang T. Differentiating between fertilized and unfertilized eggs prior to incubation based on oxygen flux measurement. Int J Agric \& Biol Eng, 2017; 10(4): 243-251.

\section{Introduction}

China has been the world leader in chicken egg production for 27 consecutive years ${ }^{[1]}$. However, hatching eggs typically have fertilization rates of $90 \%$ or less. The ability to identify the $10 \%$ of hatching eggs that are not fertilized prior to incubation would greatly increase hatching rates, lower costs and conserve

\section{Received date: 2016-05-31 Accepted date: 2017-03-01}

Biographies: Wang Qiaohua, PhD, Professor, research interest: mechatronics, intelligent detection and control, machine vision, Email: wqh@mail.hzau.edu.cn; Fu Dandan, Graduate student, research interest: intelligent detection and control, Email: fudandan@webmail.hzau.edu.cn; Zhang Tao, Graduate student, research interest: intelligent detection and control; Email: zhangtao@mail.hzau.edu.cn.

*Corresponding author: Ma Meihu, PhD, Professor, research interest: egg processing technology, College of Food Science and Technology, Huazhong Agricultural University, Wuhan 430070, China. Tel: +86-18702768307, Fax: +86-27-87283177, Email: wqh@mail.hzau.edu.cn. incubator space. Differentiating between fertilized and unfertilized eggs prior to incubation remains an unsolved challenge in the egg industry.

In the poultry industry, eggs are often subjected to candling, which involves inspecting the eggs 5-7 d after their initial incubation by passing a light through each egg. However, while candling is a simple method, it is unable to accurately detect a fertile egg within 5-7 d incubation and it cannot distinguish between the infertile egg and very early embryo mortality.

Fertilized eggs respire during incubation, the oxygen consumption of the embryo changes regularly during incubation. The entire hatching process for chicken eggs requires about $21 \mathrm{~d}$. Generally, the incubation period has two phases: initial incubation period (1-9 d) and final incubation period $(10-21 \mathrm{~d})$. In the initial incubation period, the embryo oxygen consumption changes with the incubation temperature. Increasing the environment temperature leads to an increase in oxygen 
consumption of embryo; the embryo at this stage is equivalent to a cold-blooded animal. However, in the late stage of incubation there is a different effect: when the temperature drops, the oxygen consumption is increased; at this time the embryo is equivalent to a warm-blooded animal $^{[2]}$. At low temperatures, in order to maintain metabolism, the egg needs to generate more heat which results increased oxygen consumption.

To address this issue, many native and international scholars have conducted a large number of investigations to examine the use of machine vision ${ }^{[3]}$ and hyperspectral imaging $^{[4-6]}$ for the noninvasive inspection of hatching eggs $^{[7,8]}$. These investigations have attempted to discover an egg examination method that is more effective than the currently available approaches. In particular, the use of hyper spectral imaging techniques to detect the status of hatching eggs has received a great deal of attention and has been proven to be an effective way. However, these approaches are primarily used for eggs that are in the late stages of incubation and are rarely used for the examination of eggs prior to incubation.

Given that chicken eggs are living organisms, respiration is an important physiological activity for these eggs, thus a great deal of research has been performed regarding respiration in hatching eggs ${ }^{[9,10]}$. Other investigators have examined the oxygen consumption of the chicken embryo under conditions of varying temperatures and oxygen concentrations ${ }^{[11]}$. Researchers have also used non-invasive techniques to measure the oxygen partial pressure, lateral diffusion, and chorioallantoic blood flow under the avian eggshell; these examinations revealed that the oxygen partial pressure varies in different parts of the egg, with maximal levels of oxygen partial pressure at the blunt end of the egg followed by the pointed end of the egg, and minimal levels of oxygen partial pressure near the equator of the $\operatorname{egg}^{[12]}$. The studies referenced above examine the ways in which eggs undergoing incubation are affected by their external environment and the changes that occur in the physiological parameters of these eggs. Only a few of these investigations address non-invasive approaches for differentiating between fertilized and unfertilized eggs prior to incubation.
The non-invasive micro-test technique (NMT) was originally developed by Newman and Kuhtreibe ${ }^{[13]}$. This approach is a real-time, dynamic in vivo measurement technique that provides insight into biological activities by quantifying the fluxes of ions and small molecules between a living organism and its environment. This technique allows for the detection of both the magnitude and direction of the spatial movements of these ions and small molecules without labeling or sample extraction. The NMT is primarily used in life sciences and in medicine to examine cells, including cell layers and single cells. However, although chicken eggs are live specimens, they have not been examined using this technique because of their structural peculiarities and rather large size. Thus, this article is the first investigation to use NMT to investigate gas exchange in chicken eggs and explore the differences in respiration between fertilized and unfertilized eggs. A customized NMT Egg Testing System (NMT-ETS) was constructed that allowed for the real-time monitoring of the rate and intensity of oxygen exchange between the egg and its external environment. The results revealed that prior to incubation; fertilized and unfertilized eggs exhibit significant differences in oxygen exchange rates with their surroundings.

\section{Materials and methods}

\subsection{Experimental sample}

A total of 50 fertilized eggs and 50 unfertilized eggs of the same breed, batch, and age were selected for examination in this study. The two types of eggs exhibited no significant differences in fresh weight $(p>0.05)$. The examined eggs were provided by the Beijing Huadu Yukou Poultry Company. The eggs were stored at room temperature $\left(21^{\circ} \mathrm{C}\right)$ in an environment with a relative humidity $(\mathrm{RH})$ of approximately $30 \%$.

\subsection{Determination of the fertilization status}

Fertilized and unfertilized eggs were distinguished by breaking open eggs and closely examining the shape, size, and color of the blastoderm of fertilized eggs and the blastodiscs of unfertilized eggs; this was done to determine the distinguishing physical characteristics of each type. From the cross-sectional view, the disc-shaped blastoderm is visible at the surface of the 
yolk of a fertilized egg (Figure 1a); for an unfertilized egg, there is only a white dot, the blastodisc (Figure 1b), which is lighter in shade and smaller in size than the blastoderm of a fertilized egg ${ }^{[14]}$.

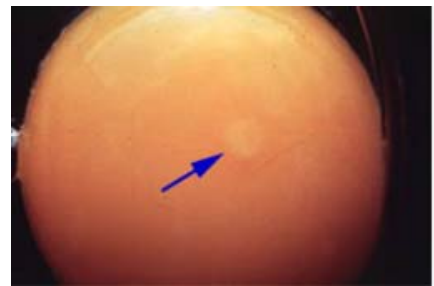

a. Blastoderm

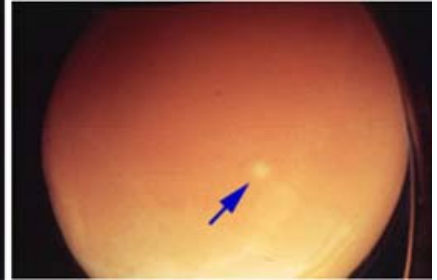

b. Blastodisc
Figure 1 Blastoderm and blastodisc of a fertilized egg

\subsection{NMT and oxygen electrodes}

2.3.1 Polarographic oxygen electrodes, calibration and testing principles

In this research, polarographic oxygen electrodes are used. A voltage of $-700 \mathrm{mV}$ was applied to each oxygen electrode as polarization voltage, which was then successively placed into three solutions with known oxygen concentrations of $0,21 \%$ and $100 \%$. Three electrical current values were detected. Using the proportional relationship between the currents and oxygen concentrations, a calibration curve was drawn, and the slope (S) of this curve was calculated ${ }^{[15]}$. A calibrated oxygen electrode was precisely placed in the NMT system near the surface (ca. $50 \mu \mathrm{m}$ from the surface) of an examined chicken egg, and current measurements were obtained at two points that were separated by a known distance $d x=300 \mu \mathrm{m}$. Data were acquired every 5-6 s over a predefined time period, and a curve depicting the temporal changes in electrical current differences was produced for each examined sample. The sign of the results indicates the direction of flux ${ }^{[16]}$. See reference $^{[17]}$ for details regarding specific testing principles.

\subsection{Methods and setups for detecting the oxygen flux}

Based on the principles that have been described above, electrical current measurements were obtained near the blunt end of the egg at two points separated by a known distance in the direction of the long axis of the egg. The difference in currents between these two points was calculated, and an automated excel spreadsheet (specialized for Flux Conversion) was used to convert this difference into an oxygen flux. The direction and magnitude of this oxygen flux was regarded as gas exchange of the egg.

The polarographic oxygen electrode non-invasive test system illustrated in the Figure 2 was used to test the flow rate of $\mathrm{O}_{2}$ through eggshell. The fertilized and unfertilized eggs were all tested in the non-invasive micro test technology of egg in this study. The test was conducted in a closed shielded environment. The shielded box is metal; it is used to prevent experiment from interference of outside static electricity and to prevent the influence of airflow. So to a certain extent it can be considered that the oxygen measured in this experiment is primarily from the respiration of eggs. The shield box is equipped with an anti-vibration table; the body-type microscope is located above the probe, so it is clear to see the eggshell surface and the probe micro-structure and to adjust the distance between the probe and the egg surface.

To perform current detection procedures, the egg was secured in an egg holder on a shockproof platform. The screen became clear by focusing on the top surface of the egg through the imaging section. The image acquisition components of the NMT-ETS (a microscope and a charge-coupled device (CCD)) were used to observe and confirm the measurement positions. The motion control component of the NMT-ETS was used to adjust the electrode to a vertical distance of $50 \mu \mathrm{m}$ above the egg surface. The probe was adjusted until the probe in the CCD imaging screen became clear. Software programs were then used for signal acquisition and processing procedures that generated continuous oxygen flux measurements. Particular emphasis was placed on preventing vibrations and shielding the NMT-ETS from electromagnetic interference during the course of the detection process ${ }^{[15]}$.

The setup of the NMT system is illustrated in the Figure 2. The detection device in the Figure is in a closed compartment, and the parameter index of this experiment was tested in a closed and shielded environment. The shielding box is metallic to prevent any interference due to static electricity which might compromise results. The experiment is carried out in a closed environment, so to a certain extent it can be 
considered that the detected oxygen using the NMT technique arise from other sources other than the test subject; therefore it can be concluded that the oxygen measured in this experiment is primarily from the respiration of the eggs.

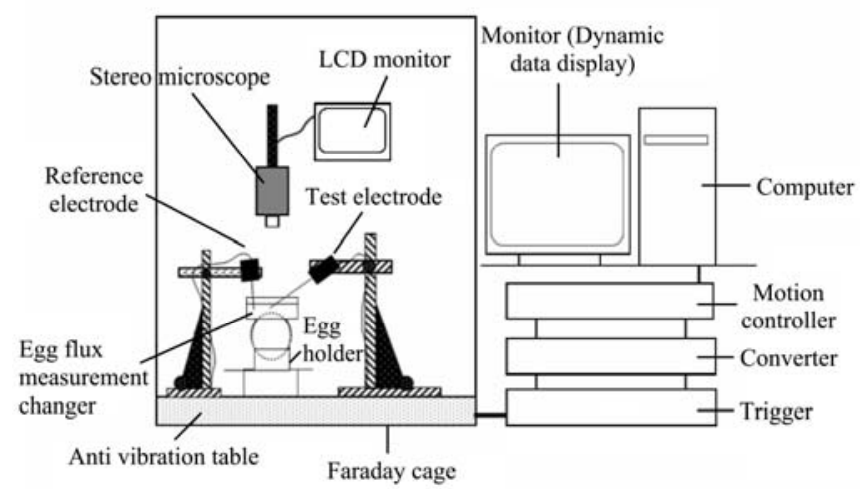

Figure 2 Setup of NMT-ETS

\subsection{Steps for detecting oxygen flux}

(1) Ten fertilized and unfertilized eggs were randomly selected for testing at 9:00 every morning. A protective cap was applied to each egg with Foamtastic plastic glue 20 min before testing.

(2) Fertilized and unfertilized eggs were successively tested, using the NMT-ETS to determine the oxygen flux near the blunt end of each egg. Oxygen consumption was most apparent at the blunt end of the tested eggs. Since this location exhibited the greatest gas exchange among the examined sites, the blunt end of the eggs was selected as the optimal test location for the subsequent experiments in this study ${ }^{[15]}$.

The oxygen microelectrode was placed $50 \mu \mathrm{m}$ above the surface of the egg. A scan testing procedure was utilized: nine points were selected for testing within a $200 \mu \mathrm{m} \times 200 \mu \mathrm{m}$ area of the blunt end of the egg, and three measurements were obtained at each of these points $^{[15]}$. Each category, i.e. fertilized and unfertilized, was tested alternatively

The eggs used in the experiments were purchased from the same farms. Fertilized eggs are selected from the farm where cocks and hens are raised together, and unfertilized eggs are selected from the farm where there are only hens. We can not guarantee that the assumed fertilized eggs are indeed fertilized, but we can guarantee that unfertilized eggs are in fact unfertilized.

(3) After each test; the fertilized and unfertilized eggs were broken and examined. The observation of a blastoderm was regarded as confirmation that an egg was fertilized. If one of these features was not evident in an allegedly fertilized egg, a replacement fertilized egg was selected and examined to ensure that the desired number of fertilized eggs was assessed in this experiment.

(4) Steps 1-3 were repeated every $2 \mathrm{~d}$ until they became addled eggs, or they collapsed, the test ended within $30 \mathrm{~d}$.

(5) The original signal collected by the electrode is an optical signal. The objective is to convert the photoelectric signals into electrical signals and record it, as shown in Figure 3. Using the Magflux conversion software, the oxygen flow rate and oxygen concentration can be retrieved, and the obtained oxygen flow rate represents the oxygen exchange between the egg and the environment.

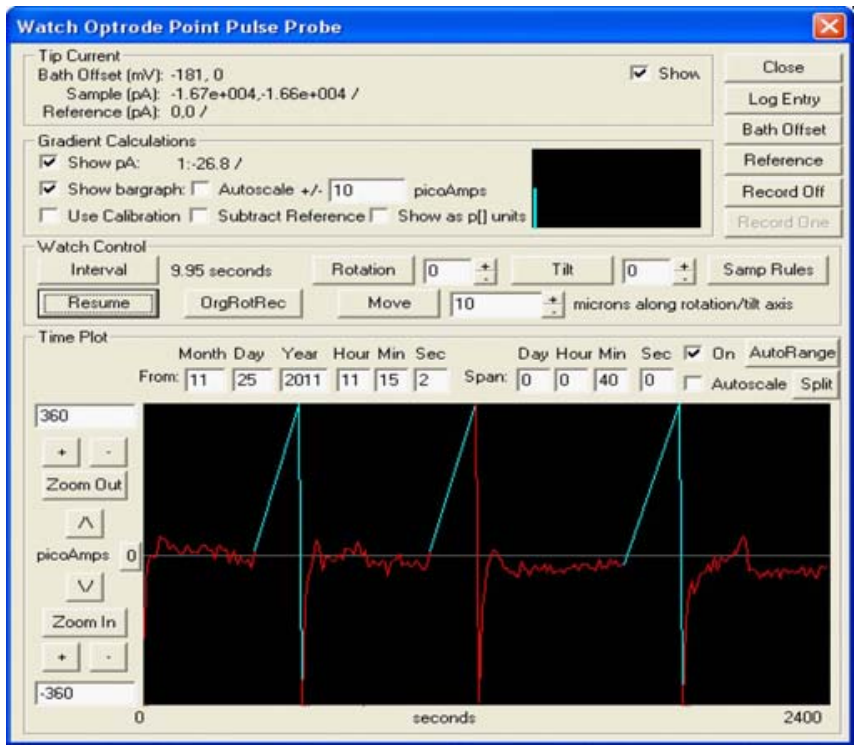

Figure 3 Date and appearance of $\mathrm{O}_{2}$ showed in flux software

\section{Results and discussion}

\subsection{Oxygen flux measurements at various heights}

To determine the optimal measurement distance, oxygen consumption was examined at several different heights above the egg surface, i.e. $50 \mu \mathrm{m}, 250 \mu \mathrm{m}$, $450 \mu \mathrm{m}, 1000 \mu \mathrm{m}$ and $3000 \mu \mathrm{m}$. The results of five examinations are depicted in Figure 4.

The five segments of each curve in Figure 4 correspond to the oxygen consumption levels that were measured at heights $50 \mu \mathrm{m}, 250 \mu \mathrm{m}, 450 \mu \mathrm{m}, 1000 \mu \mathrm{m}$, and $3000 \mu \mathrm{m}$ above the egg surface. As illustrated in the 
Figure 3, the strength of the detected signal gradually weakens as the distance of the probe from the egg surface increases. In particular, a gradient of oxygen signal magnitude exists; as the height of the probe above the egg surface increases, the probe moves along this gradient in the direction of decreasing oxygen flux. Micro-electrodes that are closer to the egg surface will more accurately approximate the egg's actual level of oxygen gas exchange and will provide stronger signal measurements. Data collected at this point will obviously be more favorable for analysis; thus, a distance of $50 \mu$ m between the electrode and the egg surface was used for subsequent experiments involving testing of both types of eggs.

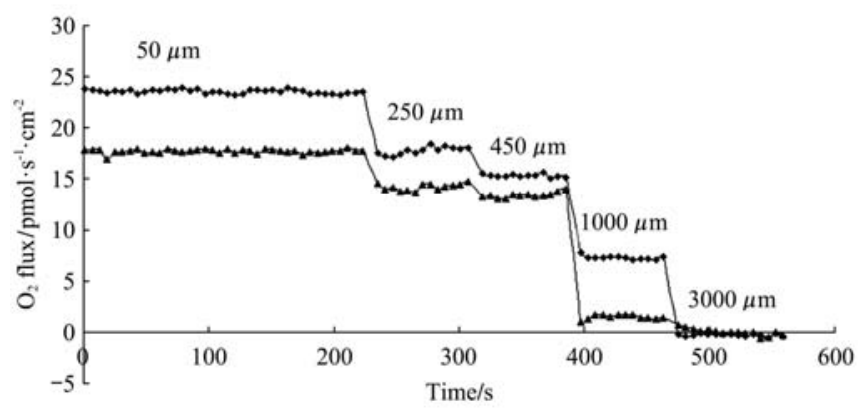

Figure 4 Oxygen consumption measured by electrodes at different heights from the egg surface

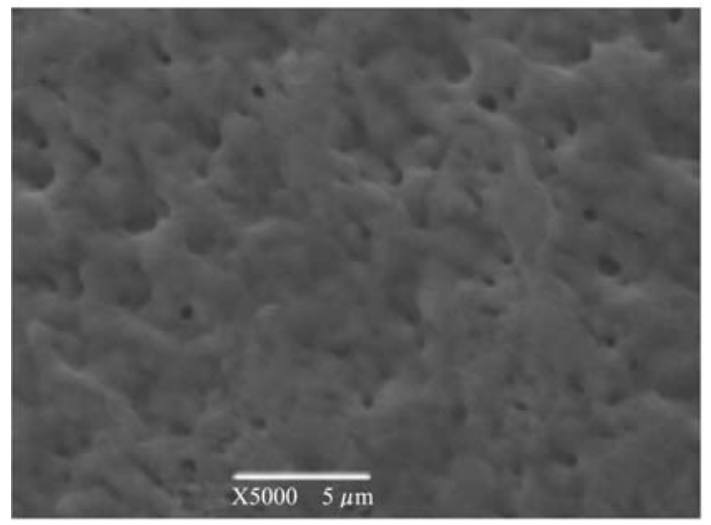

a. Original image

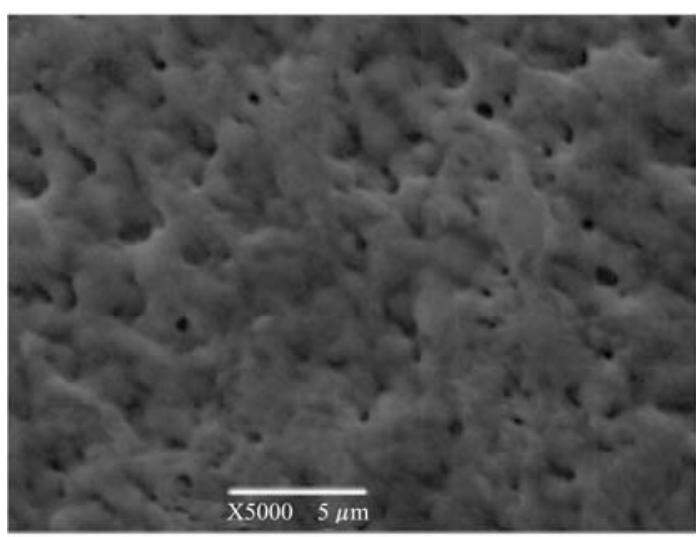

c. Opening operation and top hat transformation

\subsection{Effects of $\mathrm{O}_{2}$ flow velocity in fertilized and} unfertilized eggs eggshell ultrastructure

Observation of the eggshell surface morphology of both categories of eggs with an electron scanning microscope (X5000) was done; then a comparative analysis of the post-processed images was conducted using MATLAB. The original image of the egg shell surface ultrastructure when viewed under the electron scanning microscope is shown in Figure 5a, and its three-dimensional visual effect diagram is shown in Figure 5b. After employing MATLAB image processing techniques such as morphological image opening (imopen), smoothing transform, contrast stretch, median filter and binary processing; the entire edge was extracted by the Sobel edge detection operator. The closed circles are the shell surface pore sites (holes). By calculating the closed hole number ' $n$ ', we can know the number of holes within the image area ' $N$ ' $(N=n)^{[18]}$. The pore density of eggshell $P$ can be calculated as:

$$
P=\frac{N}{S}
$$

where, $S$ is the area of eggshell.

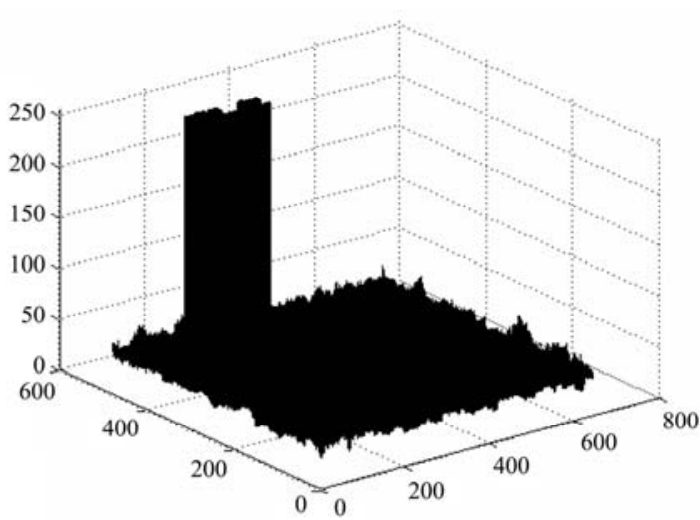

b. Three dimensional visualization effect chart of original image

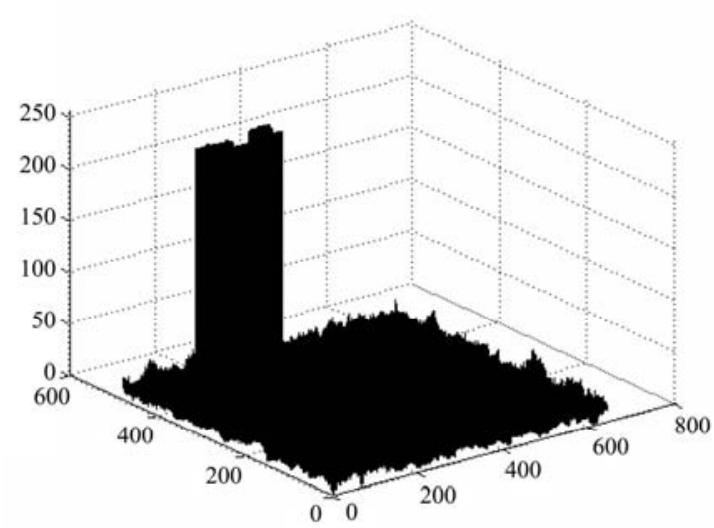

d. Three dimensional visualization effect chart of image (c) 


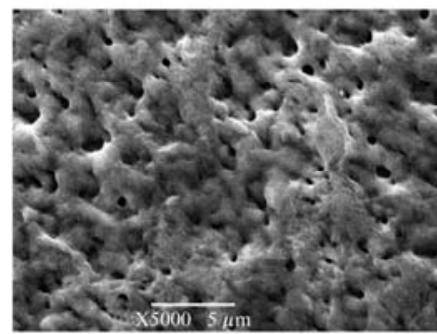

e. Contrast stretch

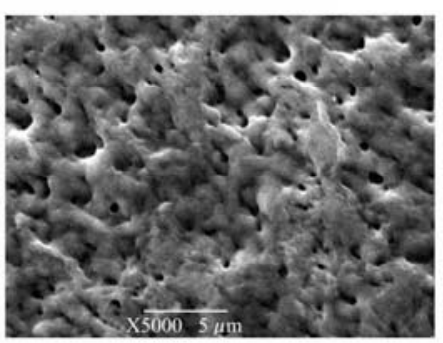

f. Median filtering

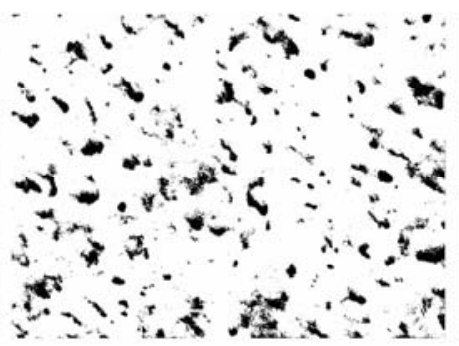

g. Binary image

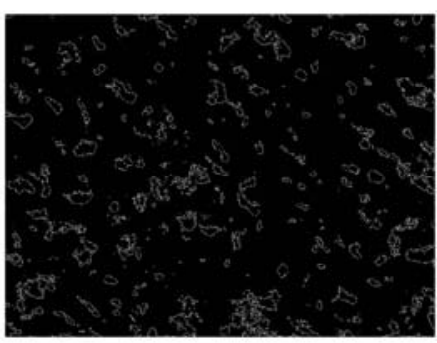

h. Edge detection by Sobel operator

Figure 5 Image processing step using MATLAB

The results are shown in Figure 6. Figures $6 \mathrm{a}, 6 \mathrm{~b}$ and $6 \mathrm{c}$ represent three kinds of the surface morphology of the shell of fertilized eggs and Figures 6c, 6d and 6e represent three kinds of the surface morphology of the shell of non-fertilized eggs. It can be seen that the shell surface of fertilized egg is relatively rough with many dense holes in its surface, the diameter of these holes is relatively large, approximately 111000 holes $/ \mathrm{mm}^{2}$. Approximately $12.5 \%$ of the surface of the fertilized eggs exhibit crack-like appearance. The shell surface of the unfertilized egg is relatively smooth, with a small hole diameter and low pore density; the density of the hole is approximately 57400 holes $/ \mathrm{mm}^{2}$. Approximately 43.8\% of the unfertilized egg shell surface shape is irregular with cracks. After conducting a statistical analysis, it can be concluded that the hole density of the fertilized eggs are significantly greater than the unfertilized eggs $(p<0.05)$.

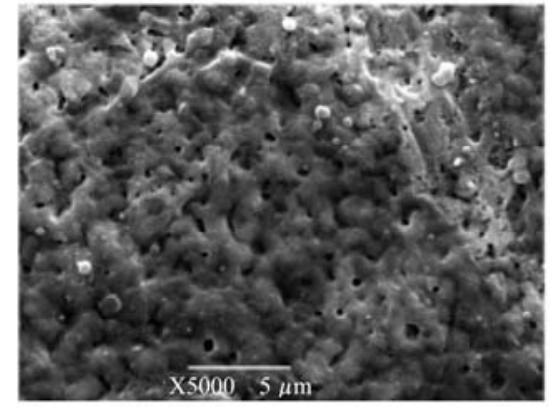

a. Fertilized egg surface ultrastructure

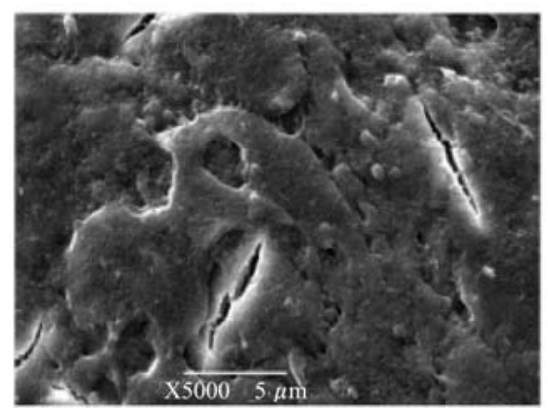

d. Non-fertilized egg surface ultrastructure

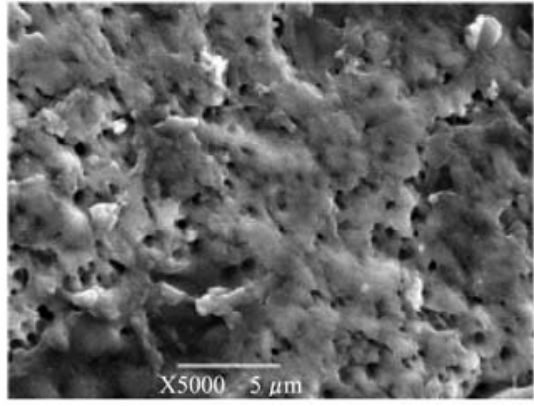

b. Fertilized egg surface ultrastructure

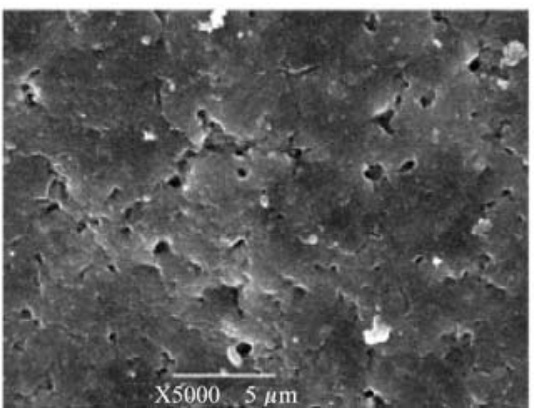

e. Non-fertilized egg surface ultrastructure

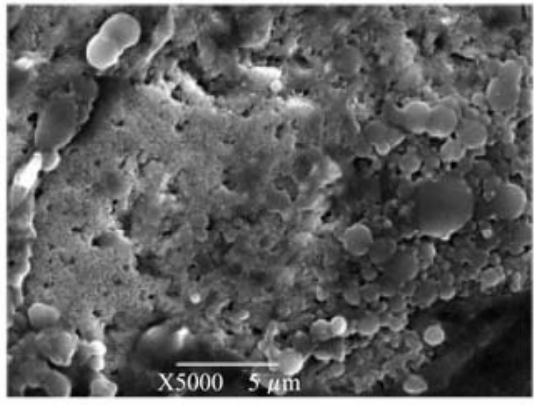

c. Fertilized egg surface ultrastructure

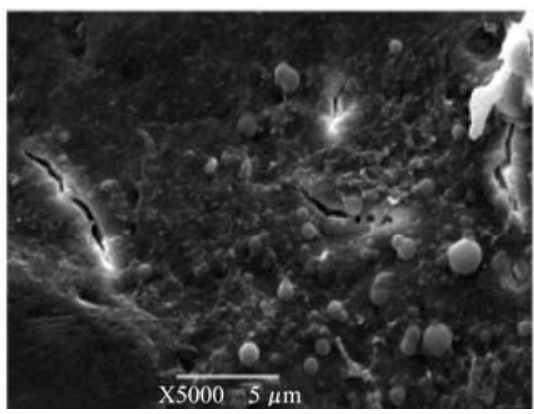

f. Non-fertilized egg surface ultrastructure

Figure 6 Surface ultra structures comparison between fertilized and non-fertilized eggs

The surface structure of the eggshell of these two categories of eggs is complex with significant differences. Further studies show that, in all cases, the rougher the surface structure of the shell, the greater the flow rate of $\mathrm{O}_{2}$. Figure 7 shows the surface structures of the shell at different flow rates, the flow rate of $\mathrm{O}_{2}$ in Figures $7 \mathrm{a}, 7 \mathrm{~b}$, 7c and $7 \mathrm{~d}$ are $45.03 \mathrm{pmol} /\left(\mathrm{cm}^{2} \cdot \mathrm{s}\right), 30.51 \mathrm{pmol} /\left(\mathrm{cm}^{2} \cdot \mathrm{s}\right)$, $18.52 \mathrm{pmol} /\left(\mathrm{cm}^{2} \cdot \mathrm{s}\right)$ and $13.07 \mathrm{pmol} /\left(\mathrm{cm}^{2} \cdot \mathrm{s}\right)$, respectively.
The reason for this is due to the varying roughness of the surface structures, the crack-like appearance and varying density of pores.

The pore density is related to the ease with which the egg exchanges gas with the environment, the more numerous the pores, the easier gas exchange will be. Based on these findings, it can be concluded that the surface structure of the egg shell has a significant impact 
on egg $\mathrm{O}_{2}$ exchange with its surrounding environment.

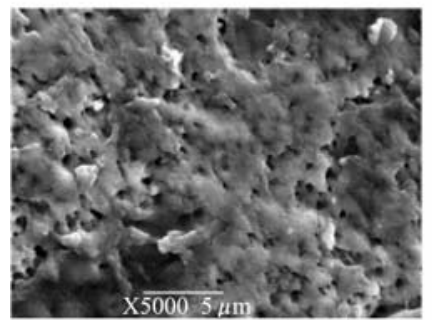

a. Eggshell surface ultrastructure at $45.03 \mathrm{pmol} /\left(\mathrm{cm}^{2} \cdot \mathrm{s}\right)$ flow rates

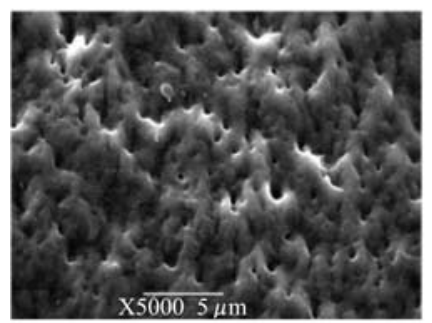

c. Eggshell surface ultrastructure at $18.52 \mathrm{pmol} /\left(\mathrm{cm}^{2} \cdot \mathrm{s}\right)$ flow rates

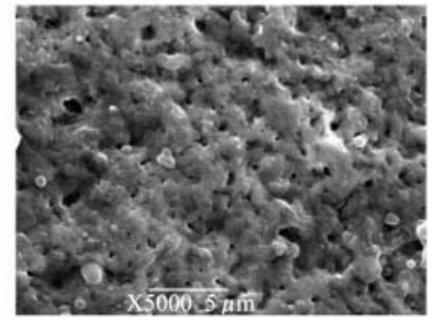

b. Eggshell surface ultrastructure at $30.51 \mathrm{pmol} /\left(\mathrm{cm}^{2} \cdot \mathrm{s}\right)$ flow rates

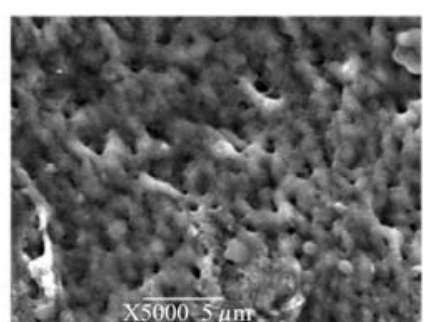

d. Eggshell surface ultrastructure at $13.07 \mathrm{pmol} /\left(\mathrm{cm}^{2} \cdot \mathrm{s}\right)$ flow rates

Figure 7 Eggshell surface ultrastructure with different respiration intensity

\subsection{Fertilized and unfertilized eggs exhibit highly} significant differences in oxygen flux levels

The results from the egg tests revealed a significant difference in respiration intensity between fertilized and unfertilized eggs; in particular, fertilized eggs demonstrate a significantly higher respiratory intensity than unfertilized eggs. This difference is shown in the Figure $8(n=6-9, p<0.01)$.

Additionally, ANOVA results show that the oxygen exchange intensities for fertilized eggs exceed $20 \mathrm{pmol} /\left(\mathrm{cm}^{2} \cdot \mathrm{s}\right)$, whereas the oxygen exchange intensities for unfertilized eggs are below this level. Thus, $20 \mathrm{pmol} /\left(\mathrm{cm}^{2} \cdot \mathrm{s}\right)$ may be regarded as a cutoff value for distinguishing between fertilized and unfertilized eggs. Differences in physiological structure may be the primary reason that fertilized and unfertilized eggs exhibit different oxygen exchange intensity levels.

(1) A fertilized egg undergoes continuous cell division within the body of a hen. Thus, this egg has already reached the blastocyst stage by the time that the egg is laid. A steady flow of energy is required for the continuing development of the embryo over time. These large energy requirements must be supported by a high level of oxygen exchange intensity and therefore a substantial oxygen flux. By contrast, an unfertilized egg may be viewed as a female germ cell or an ovum; thus,

no embryonic development or cell division occurs within an unfertilized egg, therefore unfertilized eggs require less energy than fertilized eggs ${ }^{[8]}$.

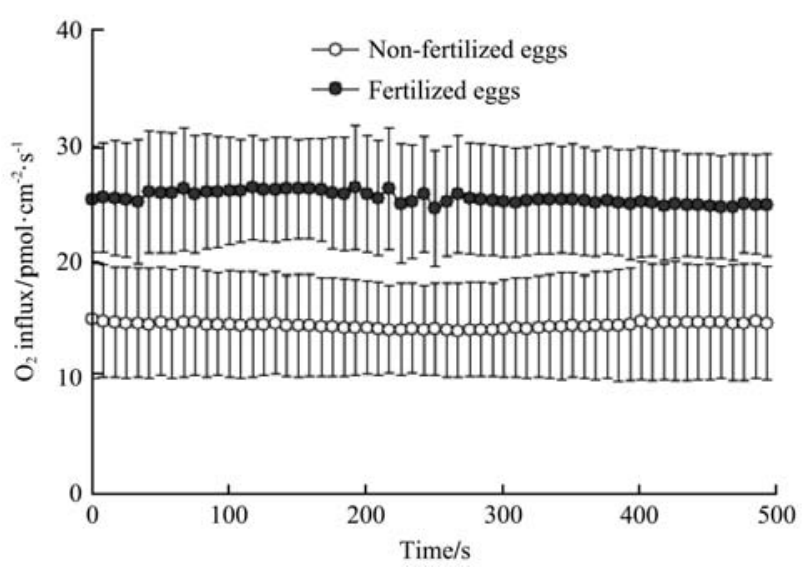

a. Oxygen fluxes of fertilized and unfertilized eggs at different time

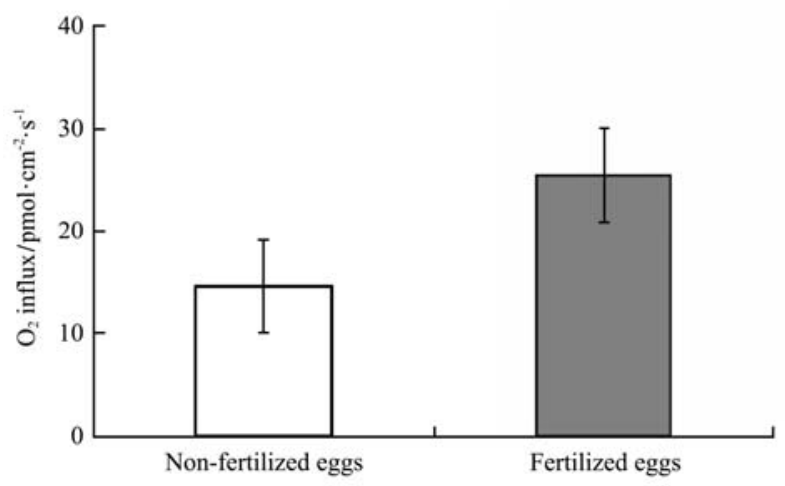

b. Average oxygen fluxes of fertilized and unfertilized eggs

Figure 8 Oxygen fluxes of fertilized and unfertilized eggs

(2) The phenomenon of higher respiration in fertilized eggs than in unfertilized eggs also relates to eggshell structure. During embryonic development, eggshell pores are utilized for gas exchange between an embryo and its external environment. Relative to unfertilized eggs, fertilized eggs feature thinner eggshells and larger and more abundant pores, as a result, relative to unfertilized eggs, fertilized eggs will demonstrate higher levels of gas exchange with their external environments.

The NMT results obtained by utilizing a polarographic oxygen electrode to measure differences in oxygen flux between fertilized and unfertilized eggs are highly consistent with the findings that would be expected from the inherent structures of these two types of eggs.

\section{Conclusions}

This study demonstrated a procedure for distinguishing 
between fertilized and unfertilized eggs prior to incubation. The intensity of oxygen exchange between chicken eggs and its surrounding environment was conclusively measured and monitored by NMT-ETS in real-time. It is proved that there exists a significant difference in oxygen flux between fertilized and unfertilized eggs. The oxygen exchange intensity of fertilized eggs exceeded $20 \mathrm{pmol} /\left(\mathrm{cm}^{2} \mathrm{~s}\right)$, whereas the oxygen exchange intensity of unfertilized eggs was below this value. The method used in this study shows that the oxygen flux of an egg can be determined in approximately 1-2 min, allowing for the rapid assessment of the fertilization status of eggs prior to incubation. The results showed that the oxygen flux measurement model as method for categorizing fertile versus infertile eggs is a novel, efficient way to differentiate between fertilized and unfertilized chicken eggs prior to incubation. This study has drawn the following conclusions:

(1) The egg absorbs oxygen, as shown by the oxygen flux detected at different distances from the surface of both categories of eggs.

(2) The surface structure of the egg shell has a significant impact on $\mathrm{O}_{2}$ exchange rate with surrounding environment. The surface structure of fertilized and unfertilized eggshells are complex with significant differences $(p<0.05)$. The surface of the fertilized eggshell is rougher, and its surface holes are larger and more numerous, this facilitates easier gas exchange with its surrounding environment.

(3) The difference in oxygen flux between fertilized and unfertilized eggs is highly significant $(p<0.01)$.

(4) Oxygen flux detection can be used to determine whether an egg is fertilized or unfertilized prior to incubation. Further research could improve the test methods and means suggested in this article and implement this technology in practice.

\section{Acknowledgments}

We thank the Younger USA (Xuyue Beijing) NMT Service Center and the Beijing Huadu Yukou Poultry Company for their strong support for this study. This work was supported by National Natural Science
Foundation of China (31371771), Special Fund for Agroscientific Research in the Public Interest (201303084) and National Science and Technology Support Programme (2015BAD19B05).

\section{[References]}

[1] Ma M H. Processing technology and quality safety control strategy of egg products. China Poultry, 2009; 31: 1-5. (in Chinese)

[2] Joseph M M. Factors affecting the hatchability and hatching. China Poultry, 2002; 24: 27-32. (in Chinese)

[3] Das K, Evans M D. Detecting fertility of hatching eggs using machine vision II: Neural network classifiers. Transactions of the ASAE, 1992; 35: 2035-2041.

[4] Bamelis F R, Tona K, de Baerdemeaker J G. Detection of early embryonic development in chicken eggs using visible light transmission. British Poultry Science, 2002; 43: 204-212.

[5] Smith D P, Lawewnce K C, Park B. Detection of fertility and early development of hatching eggs with hyperspectral imaging. Proc. 11th European Symposium on the Quality of Eggs and Egg Products Netherlands: World's Poultry Science Association, 2005; pp.176-180.

[6] Jones S T, Shattuck R E. Detection of Early Embryonic Development in hatching eggs: a hyperspectral imaging systems and neural network approach. Johns Hopkins Applied Physics Laboratory Technical Digest, 2005; 1: 67-73.

[7] Bamelis F, Kemps B, Mertens K, Tona K, de Ketelaere B, Decuypere E, et al. Non-destructive measurements on eggs during incubation. Avian and Poultry Biology Reviews, 2004; 15: 3-4.

[8] Liu L, Ngadi M. Detecting fertility and early embryo development of chicken eggs using near-infrared hyper-spectral imaging. Food and Bio-process Technology, 2013; 6: 9.

[9] Kerstin W A. Seymour R S. Effect of regional changes to shell conductance on oxygen consumption and growth of chicken embryos. Respiration Physiology, 2002; 129(3): 385-395.

[10] Sunder L M,Tompkins D M, Hudson P J. The role of oxygen availability in the embryo nation of Heterakis gallinarum eggs. International Journal for Parasitology, 2000; 30: 1481-1485.

[11] Pearson $\mathrm{J}$ T, Haque $\mathrm{M}$ A, Hou $\mathrm{P}$ C, Tazawa $\mathrm{H}$. Developmental patterns of $\mathrm{O}_{2}$ consumption, heart rate and $\mathrm{O}_{2}$ pulse in unturned eggs. Respiration Physiology, 1996; 103: 83-87.

[12] Seymour R S, Kerstn W A. Non-invasive measurement of 
oxygen partial pressure, lateral diffusion and chorioallantoic blood flow under the avian eggshell. Comparative Bio-chemistry and Physiology, Part A, 2008; 150: 258-264.

[13] Kuhtreiber W M, Laffe L F. Detection of extracelluar calcium gradients with a calcium-specific vibrating electrode. Journal of Cell Biology, 1990; 110: 1565-1573.

[14] Bakst M R, Gupta S K, Potts W, Akuffo V. Gross appearance of the turkey blastoderm at oviposition. Poultry Science, 1998; 77(8): 1228-1233.

[15] Wang Q H, Zhang T, Ma M H. Oxygen respiratory regularity of eggs in storage period monitoring by non-invasive micro-test technique. Transactions of the CSAE, 2014; 3(5): 255-261.
[16] Chwalibog A, Tauson A H, Ali A, Matthiesen C, Thorhayge K, Thorbek G. Gas exchange, heat production and oxidation of fat in chicken embryos from a fast or slow growing line. Comparative Biochemistry and Physiology, Part A, 2007; 146: 305-309.

[17] Jacopo P M, Katherine L. Oxygen consumption of the chicken embryo: interaction between temperature and oxygenation, science direct. Respiratory Physiology \& Neurobiology, 2005; 146: 97-106.

[18] Wang Q H, Zhang T, Ma M H, Li X M. Study on the Relationship between egg $\mathrm{O}_{2}$ respiration and eggshell ultra structure. Advance Journal of Food Science and Technology, 2015; 9(3): 159-166. 